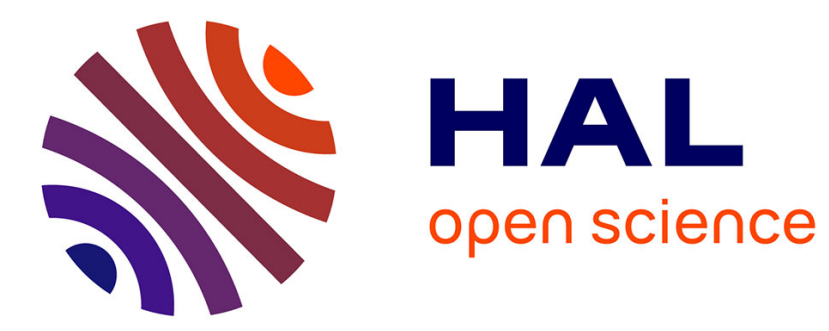

\title{
Geminate carbon monoxide rebinding to a c-type haem
}

G. Silkstone, Audrius Jasaitis, Marten H. Vos, M. Wilson

\section{To cite this version:}

G. Silkstone, Audrius Jasaitis, Marten H. Vos, M. Wilson. Geminate carbon monoxide rebinding to a c-type haem. Dalton Transactions, 2005, 21 (7), pp.3489. 10.1039/B508183C . hal-00829233

\section{HAL Id: hal-00829233 \\ https://hal-polytechnique.archives-ouvertes.fr/hal-00829233}

Submitted on 19 May 2014

HAL is a multi-disciplinary open access archive for the deposit and dissemination of scientific research documents, whether they are published or not. The documents may come from teaching and research institutions in France or abroad, or from public or private research centers.
L'archive ouverte pluridisciplinaire HAL, est destinée au dépôt et à la diffusion de documents scientifiques de niveau recherche, publiés ou non, émanant des établissements d'enseignement et de recherche français ou étrangers, des laboratoires publics ou privés. 


\title{
Geminate carbon monoxide rebinding to a $c$-type haem $\dagger$
}

\author{
G. Silkstone, ${ }^{* a}$ A. Jasaitis, ${ }^{b}$ M. H. Vos ${ }^{b}$ and M. T. Wilson ${ }^{a}$ \\ ${ }^{a}$ University of Essex, Colchester, UK CO4 3SQ.E-mail: silkgi@essex.ac.uk; \\ Tel: +44 1206872119 \\ ${ }^{b}$ Ecole Polytechnique-ENSTA, 91128 , Palaiseau Cedex, France
}

A chemically modified form of cytochrome $c$ (cyt. $c$ ), termed carboxymethyl cytochrome $c$ (cm cyt. $c$ ), possesses a vacant sixth coordination site to the haem iron that is available to bind external ligands. We present data on the rapid flash photolysis of $\mathrm{CO}$ from the ferrous haem iron of cm cyt. $c$ and describe the kinetics and spectral transitions that accompany the recombination. This was achieved using 30 -femtosecond laser pulses and a white light continuum to monitor spectral transitions. Whereas the photo-dissociation quantum yield is close to 1 , the yield of $\mathrm{CO}$ escape from the protein (the apparent quantum yield, $\varphi$ ) relative to myoglobin $(\varphi=1)$ is small due to rapid geminate recombination of $\mathrm{CO}$. On ligand photo-dissociation the haem undergoes a spin-state transition from low-spin ferrous $\mathrm{CO}$ bound to penta-coordinate high-spin. Subsequently the system reverts to the $\mathrm{CO}$ bound form. The data were fitted with a minimum number of exponentials using global analysis. Recombination of $\mathrm{CO}$ with the haem iron of $\mathrm{cm}$ cyt. $c$ is multiphasic ( $\tau=16 \mathrm{ps}, 120 \mathrm{ps}$ and $1 \mathrm{~ns})$, involving three spectrally distinct components. The fraction of haem (0.11) not recombining with $\mathrm{CO}$ within $4 \mathrm{~ns}$ is similar to the value of $\varphi(0.12)$ measured on the same preparation by the "pulse method" (M. Brunori, G. Giacometti, E. Antonini and J. Wyman, Proc. Natl. Acad. Sci. USA, 1973, 70, 3141-3144, ref. 1). This implies that no further geminate recombination occurs at $t>4 \mathrm{~ns}$. This unusually efficient CO-haem geminate recombination indicates the sterically hindered ("caged") nature of the distal haem pocket in $\mathrm{cm}$ cyt. $c$ from which it is difficult for $\mathrm{CO}$ to escape. The large geminate phase may be contrasted with the behaviour of myoglobin in which geminate recombination is small. This is in general agreement with the well-documented extensive structural dynamics in myoglobin that allow ligand passage, and a higher structural rigidity in cyt. $c$ imposed by the restraints of minimising reorganisation energy for electron transfer (M. Brunori, D. Bourgeois and D. Vallone, J. Struct. Biol., 2004, 147, 223-234, ref. 2). The high pH ferrous form of cm cyt. $c$ is a low-spin species having a lysine bound to the central iron atom of the haem (M. Brunori, M. Wilson and E. Antonini, J. Biol. Chem., 1972, 247, 6076-6081; G. Silkstone, G. Stanway, P. Brzezinski and M. Wilson, Biophys. Chem., 2002, 98, 65-77, refs. 3 and 4). This high $\mathrm{pH}(\mathrm{pH} \sim 8)$ form of deoxy $\mathrm{cm}$ cyt. $c$ undergoes photo-dissociation of lysine (although the proximal histidine is possible) after photo-excitation. Recombination occurs with a time constant $(\tau)$ of $\sim 7 \mathrm{ps}$. This is similar to that observed for the geminate rebinding of the Met80 residue in native ferrous cyt. $c(\tau \sim 6 \mathrm{ps})$ following its photo-dissociation (S. Cianetti, M. Negrerie, M. Vos, J.-L. Martin and S. Kruglik, J. Am. Chem. Soc., 2004, 126, 13 932-13 933; W. Wang, X. Ye, A. Demidov, F. Rosca, T. Sjodin, W. Cao, M. Sheeran and P. Champion, J. Phys. Chem., 2000, 104, 10 789-10 801, refs. 5 and 6).

\section{Introduction}

Redox reactions are at the heart of numerous processes of biological importance. ${ }^{7}$ The theoretical framework provided by Marcus, ${ }^{8}$ and the availability of three-dimensional structures of many redox proteins have placed studies of electron transfer on a firm footing allowing predictions to be made and experimentally tested. For non-naturally light-activated systems, such studies often rely on techniques such as stopped-flow spectroscopy or methods in which electron transfer is initiated by a laser pulse. Both stopped-flow and flash methods have advantages but also suffer severe limitations. While flow methods have the advantage that reactions between in vivo redox partners may be studied without recourse to protein modification they suffer from the disadvantage that the true electron transfer rate constant $\left(k_{\mathrm{et}}\right)$ may not be measured directly. This is because $k_{\text {et }}$ is generally much larger (up to $10^{5} \mathrm{~s}^{-1}$ ) than the pseudofirst order rate for complex formation and, in any case, is often too large to be determined with instruments having a dead time of $\sim 1 \mathrm{~ms}$. Similarly, laser pulse methods, while having sufficient time resolution to determine ket have several important disadvantages. First, they often rely on chemical coupling of a light sensitive electron donor to one of the partners that may perturb the system. ${ }^{9}$ Secondly, because the excited state

\footnotetext{
$\dagger$ Based on the presentation given at Dalton Discussion No. 8, 7-9th September 2005, University of Nottingham, UK.
}

of the donor is generally very short lived, the quantum yield of the process i.e. electron delivered to acceptor per pulse, is generally very low $(\sim 0.05) .^{10,11}$

Our objective has been to develop a method to study interprotein electron transfer that combines the advantages of the standard techniques while largely overcoming the drawbacks. Our method employs proteins in "quasi-native" states and, while not requiring chemical coupling of a light inducible reductant, delivers electrons very rapidly on laser activation. In addition it has the potential for delivering one electron for each $\mathrm{CO}$ dissociated, a much higher electron stoichiometry than usually available. The general principle of the method depends upon engineering a $\mathrm{CO}$ binding site into a redox haem protein (the electron donor) and relies on the fact that $\mathrm{CO}$ has a high affinity for ferrous haem proteins but does not bind to the ferric haem counterpart. Invariably CO complexes of ferrous haem proteins are light sensitive, such that a brief light pulse, e.g. from a laser, will dissociate the $\mathrm{CO}$ from the haem group, leaving this as a five coordinate high-spin ferrous species. Five coordinate haem proteins generally have a lower redox potential than their hexacoordinate forms and thus photo-dissociation of $\mathrm{CO}$ has the effect of rapidly switching the redox potential of the protein, changing a good electron acceptor (the $\mathrm{CO}$ adduct) into a good electron donor (five coordinate ferrous haem). Thus, having mixed the $\mathrm{CO}$ adduct of such a haem protein with a redox partner and allowed time for a complex to form one may initiate electron transfer at will with a laser. We have exploited this 
strategy in flow-flash experiments using a modified form of cyt. $c$ termed $\mathrm{cm}$ cyt. $c$, in which the iron is penta-coordinate due to carboxymethylation of the methionine- 80 that normally fills the sixth coordination site in the native protein. Mixing the CO complex of cm cyt. $c$ with a suitable redox active-partner produces a protein complex which, however, does not undergo electron transfer as the ferrous form of cm cyt. $c$ is stabilised by the bound ligand. Laser photolysis of $\mathrm{CO}$ from $\mathrm{cm}$ cyt. $c$ renders this penta-coordinate high spin, but now with the potential to donate electrons.

Our studies on cyt. $c$ oxidase and plastocyanin have provided proof of principle for the method but the use of cm cyt. $c$, although useful, is not ideal and we have now produced a set of genetically engineered Met80X mutants that bind $\mathrm{CO}$ and are light sensitive. Unfortunately these proteins have very low quantum yields and are unsuitable for the use described. ${ }^{4}$ In order to understand why this is so and thus to help further design of mutants we have undertaken a set of experiments to study the events following laser excitation. In this paper we describe our initial studies using femtosecond laser photolysis of the $\mathrm{CO}$ adduct of cm cyt. $c$.

From another perspective, we note that in contrast to the highaffinity haem ligand NO, efficient geminate haem-CO rebinding in proteins is unusual. The present system gives the unique opportunity to study parameters that determine $\mathrm{CO}$ binding to haem

\section{Materials and methods}

\section{Cm cyt. $c$ preparation}

$\mathrm{Cm}$ cyt. $c$ was prepared by treatment of native horse heart cyt. $c$ (Sigma) with potassium cyanide $(\mathrm{KCN})$ and bromoacetic acid (BrAc) (see Scheme 1). The cm cyt. $c$ preparation was adapted from the method of Schejter and George, but with a minimum incubation time of cyt. $c$ with the KCN and BrAc. ${ }^{12}$ Approximately $10 \mathrm{mg}$ of horse heart cyt. $c$ was dissolved into $1 \mathrm{ml}$ of buffer (100 mM phosphate, pH 7.0) with stirring ([protein] $\sim 1 \mathrm{mM}$ ). A $0.4 \mathrm{M} \mathrm{KCN}$ solution was carefully prepared by dissolving solid $\mathrm{KCN}$ in buffer $(100 \mathrm{mM}$ borate, $\mathrm{pH} 9.0)$ and ensuring the $\mathrm{pH}$ did not fall below neutrality by adding small amounts of $1 \mathrm{M} \mathrm{NaOH}$ and that the final $\mathrm{pH}$ was 7.0. The $\mathrm{KCN}$ solution $(2 \mathrm{ml})$ was added to the protein solution. Fig. 1 shows the nmr structure of the cyanide adduct of native horse heart ferri cyt. $c$ solved by Yao et al. (see Discussion).$^{13} \mathrm{~A}$ $0.4 \mathrm{M}$ BrAc solution was prepared by dissolving solid BrAc in buffer (100 mM phosphate, $\mathrm{pH} 7.0)$ and the $\mathrm{pH}$ was adjusted to $\sim 7.0$ by adding $\mathrm{NaOH}$. This BrAc solution was added to the protein/KCN solution to give a total reaction mixture
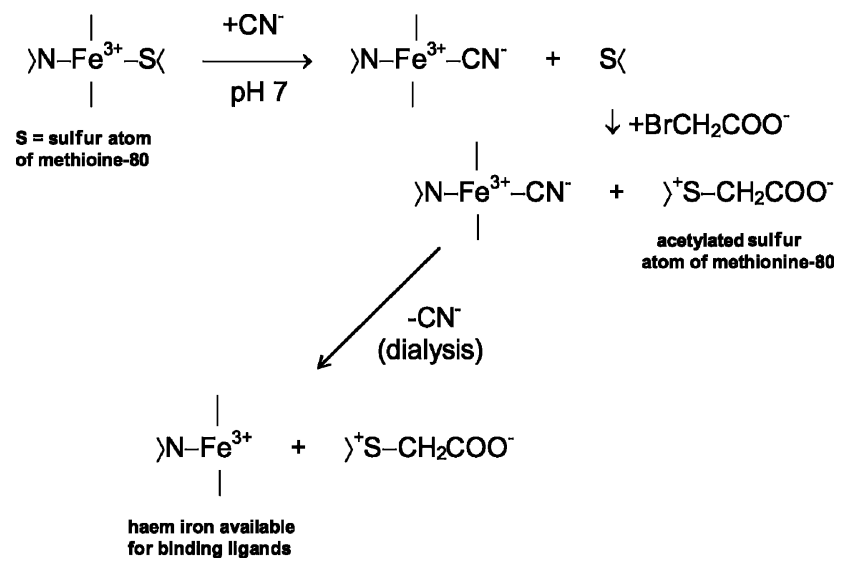

Scheme 1 The reaction of cyt. $c$ with $\mathrm{CN}^{-}$and bromoacetic acid to form $\mathrm{cm}$ cyt. $c$. Acetylation of the sulfur atom of methionine- 80 renders it unable to bind to the sixth coordination site of the haem iron. The haem iron of cm cyt. $c$ is available to bind external (e.g. $\mathrm{CO}$ ) and internal (e.g. lysine) ligands.

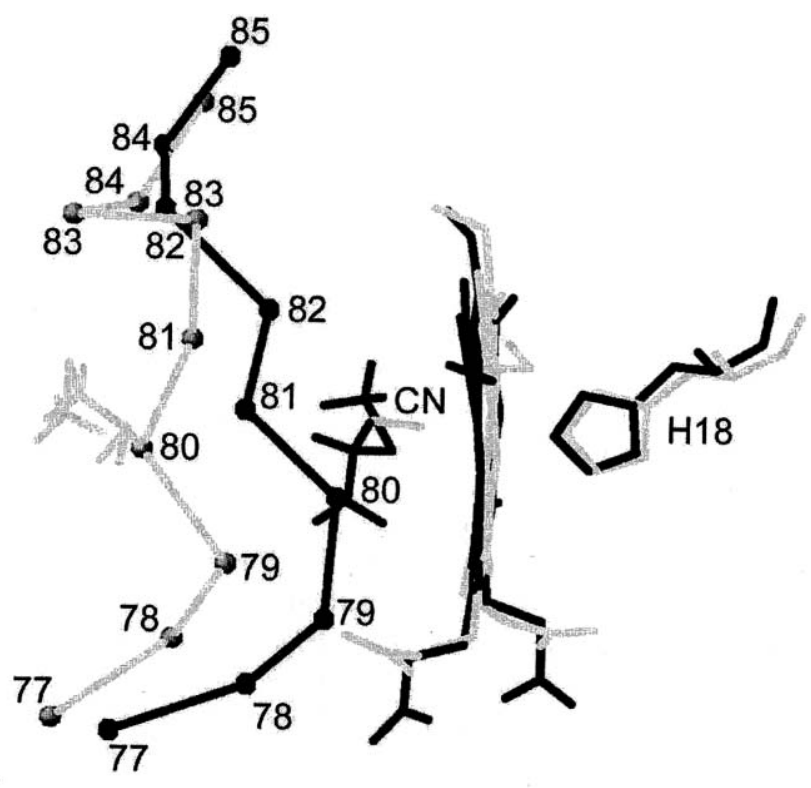

Fig. 1 The secondary structure (NMR) of the $\mathrm{CN}^{-}$adduct of horse heart ferri cyt. $c$, the rearrangement of backbone residues 77-85. Native horse heart ferri cyt. $c$, is represented by black and the $\mathrm{CN}^{-}$adduct by light grey. ${ }^{13}$ (Reproduced with permission from J. Biol. Inorg. Chem., (C) 2002).

volume of $\sim 5 \mathrm{ml}$, equimolar in the modifying reagents $\mathrm{KCN}$ and $\mathrm{BrAc}$ and $\sim 200 \mu \mathrm{M}$ in protein. The solution was stirred at room temperature, and at half hour intervals a small aliquot $(\sim 10 \mu \mathrm{l})$ was removed to monitor the progress of the reaction. To the aliquot was added $\sim 1 \mathrm{ml}$ of buffer ( $100 \mathrm{mM}$ phosphate, $\mathrm{pH}$ 7.0) and a few grains of solid dithionite was added and pure $\mathrm{CO}$ bubbled into the solution. Any cyt. $c$ that had undergone carboxymethylation of the sulfur atom of Met 80 was reduced to the ferrous form (thereby displacing the $\mathrm{CN}^{-}$from the iron) and combined with $\mathrm{CO}$ to give the ferrous $\mathrm{CO}$ adduct (CO-cm cyt. $c$ ), identified by its distinct absorption maximum in the Soret region at $414 \mathrm{~nm}\left(\varepsilon \approx 219000 \mathrm{M}^{-1} \mathrm{~cm}^{-1)} .^{3}\right.$ The reaction was stopped once all the cyt. $c$ was converted $(\sim 4-6 \mathrm{~h})$ by passing the mixture down a column containing Sephadex G25 pre-equilibrated with buffer (100 mM phosphate, $\mathrm{pH} 7.0)$, this separated the KCN and $\mathrm{BrAc}$ in solution from the $\mathrm{cm}$ cyt. $c$. Following passage through the column the cm cyt. $c$ was extensively dialysed against buffer (100 mM phosphate, $\mathrm{pH}$ 7.0) to remove cyanide bound to the ferric form.

Analysis of the product by mass spectroscopy (MALDI-ToF, Bruker Daltonics/Reflex IV) showed two forms of cm cyt. $c$ differing in mass from the native protein by +58 and $+113 \mathrm{Da}$. This closely approximates to the addition of one $(+58)$ or two $(+116)$ acetyl groups. One of these must be to Met80, as all the protein bound $\mathrm{CO}$, the other is most likely to be to Met65 although other residues can be modified. This heterogeneity is in our view not responsible for the kinetics we observe and reasons for this view will be discussed later.

\section{Sample preparation}

For the spectroscopic measurements, cm cyt. $c$ was prepared to a haem concentration of $\sim 50 \mu \mathrm{M}$ in a gas tight optical cell with an optical path length of $1 \mathrm{~mm}$. For the deoxy form of cm cyt. $c$, the de-gassed as-prepared (ferric) sample was reduced with $1 \mathrm{mM}$ sodium dithionite. For the $\mathrm{CO}$ form of $\mathrm{cm}$ cyt. $c$, the deoxy form was equilibrated with $1 \mathrm{~atm}(1 \mathrm{~atm}=101.3 \mathrm{kPa})$ $\mathrm{CO}$. The buffer used in all experiments (unless otherwise stated) was Hepes (20 mM, pH 8.0). 


\section{Spectroscopy}

Steady-state spectra were recorded using a Shimadzu UV-Vis 1601 or a Cary 5E spectrophotometer. Spectra were unchanged after the femtosecond laser flash experiments. Multicolour femtosecond absorption spectroscopy ${ }^{14}$ was performed with a 30fs pump pulse centered at $565 \mathrm{~nm}$ and a $<30$ fs white light continuum probe pulse, at a repetition rate of $30 \mathrm{~Hz}$. Full spectra of the test and reference beams were recorded using a combination of a polychromator and a CCD camera. All experiments were carried out at $21{ }^{\circ} \mathrm{C}$. The sample was continuously moved perpendicular to the beams to ensure sample renewal between shots.

Basic data matrix manipulations and presentation were performed using Matlab (The Mathworks, South Natick, MA). The absorbance changes were treated using the SPLYMOD algorithm, ${ }^{15}$ with a Matlab interface. ${ }^{16}$ The geminate $\mathrm{CO}$ recombination data was also analysed using the maximum entropy method (MEM). ${ }^{17}$ The MEM algorithm describes the data by a distribution of rate constants with a minimal number of peaks as justified by the data's signal-to-noise.

The quantum yield of CO-cm cyt. $c$ was also measured by the "pulsed" method developed by Brunori and co-workers, ${ }^{1}$ and described previously. ${ }^{4}$ Lamp flash photolysis was performed using an Applied Photophysics instrument equipped with a xenon flash (instrument dead time $0.2-1 \mathrm{~ms}$ depending on flash intensity). The flash intensity was attenuated using a set of neutral density filters. The monitoring beam was attenuated using neutral density filters so as to minimise $\mathrm{CO}$ photolysis prior to flash, and myoglobin $(\varphi=1)$ was used as a calibrant protein. The difference spectra, $\mathrm{CO}-\mathrm{cm}$ cyt. $c$ minus ferrous $\mathrm{cm}$ cyt. $c$ was measured by steady state. The same sample was then flash photolysed in the lamp flash instrument at many values of single wavelength in order to reconstruct another difference spectra (flash constructed. The CO recombination following flash was monitored at $414 \mathrm{~nm}$ (the Soret maxima for CO-cm cyt. $c$ ). The quantum yield was determined by taking the absorbance change following flash as a fraction of the total absorbance change observed for the steady state difference spectra at a given wavelength (in most cases $414 \mathrm{~nm}$ ). The $\mathrm{CO}$ concentration was kept quite low (commonly $\sim 50 \mu \mathrm{M}$ ) so that the rate of $\mathrm{CO}$ recombination from bulk was not "lost" in the dead time of the instrument.

\section{Results}

\section{Cm cyt. $c-\mathrm{CO}$}

The CO adduct of ferrous cm cyt. $c$ was found to be light sensitive, as expected from the behaviour of other ferrous haem proteins, and photo-excitation led to dissociation of $\mathrm{CO}$ from the haem. In Fig. 2 (panel A), we present transient absorption spectra that represent the difference spectra $\left(\mathrm{Fe}^{2+}\right.$ minus $\mathrm{Fe}^{2+}$ $\mathrm{CO})$ recorded at selected delay times after photo-excitation. The spectra reflect a substantial red shift of the Soret band as expected for a five-coordinate minus six-coordinate ferrous haem spectrum, and are qualitatively similar to the spectra associated with $\mathrm{CO}$ dissociation from other haem proteins, and in particular also to those associated with methionine dissociation from native cyt. $c .^{6}$ Therefore, the spectra are provisionally assigned to formation of five-coordinate haem. The difference spectrum diminishes as $\mathrm{CO}$ recombines and the time scale for recombination indicates that the majority of this reaction occurs in a very rapid geminate process. A multiexponential decay model yields three relaxation processes $(\tau=$ 16, 120 and 1000 ps), see Fig. 2 (panel B). Analysis of geminate $\mathrm{CO}$ recombination using MEM, also suggests that $\mathrm{CO}$ rebinding is not a gradual distribution of phases but consists of three distinctive exponential processes ( $\tau=3,68$ and 600 ps, see Fig. 2, panel C). The values found for the time constants are comparable given that the MEM analyses kinetics at a single wavelength
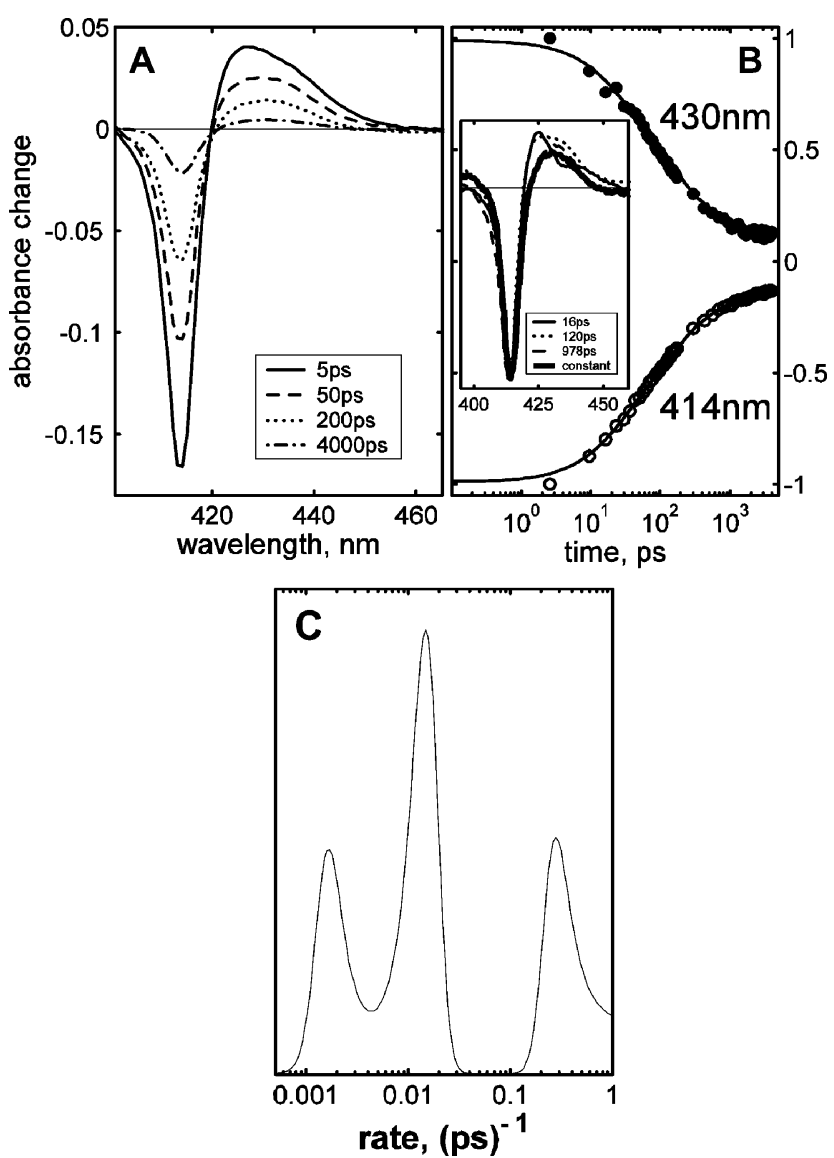

Fig. 2 Kinetics of $\mathrm{CO}$ recombination to $\mathrm{cm}$ cyt. $c$ following photo-dissociation. Panel A: Transient spectra at selected delay times after photolysis. Panel B: Time courses monitored at 430 and $414 \mathrm{~nm}$ (solid line is a theoretical curve according to parameters from the fit); Inset: the spectral components from the multi-exponential global fit (DAS components) and the final spectrum (constant, at $4 \mathrm{~ns}$ ). The relative amplitude of each component (normalized to the photolysis) is 0.27 (16 ps), 0.45 (120 ps), 0.17 (1 ns) and 0.11 (constant). Panel C: Results from the analysis of the CO rebinding kinetics at $430 \mathrm{~nm}$ using the MEM.

while the global fit analyses the whole spectrum. The spectral characteristics of the decay phases are quite distinct (Fig. 2, inset to panel B). The relative amplitude of each component (normalised to 1 at the bleaching at $414 \mathrm{~nm}$ ) is 0.27 (16 ps), 0.45 $(120 \mathrm{ps}), 0.17(120 \mathrm{ps})$ and 0.11 (constant at $4 \mathrm{~ns})$. The latter relative amplitude of 0.11 represents the percentage $(11 \%)$ of $\mathrm{CO}$ that does not recombine with the haem iron during the first $4 \mathrm{~ns}$ following photo-dissociation. This value is consistent with the measured apparent quantum yield of $\mathrm{CO}$ release from the protein (see below).

\section{Cm cyt. $c$ (deoxy)}

Photolysis of the protein at $\mathrm{pH} 8$ in the absence of added $\mathrm{CO}$ led to the appearance of a rapid transient. In Fig. 3 (panel A), we present transient absorption spectra recorded at selected delay times after photo-excitation that we assign to the difference spectrum $\mathrm{Fe}^{2+}$ minus $\mathrm{Fe}^{2+}-\mathrm{L}$, where $\mathrm{L}$ is an intrinsic amino acid other than methionine (proximal His18 or distal Lys79). This difference spectrum bleaches as the intrinsic ligand recombines and the time scale indicates that this reaction occurs in a very fast geminate process. Analysis of the transient spectra yields a single picosecond decay phase ( $\tau \sim 7$ ps), see Fig. 3 (panel B).

\section{Measurement of apparent quantum yield $(\varphi)$ for $\mathrm{cm} c y t . c$ by the "pulsed" method}

The apparent quantum yield was measured at pH 7.0. In Fig. 4 we present the spectra for the ferrous and ferrous-CO forms 


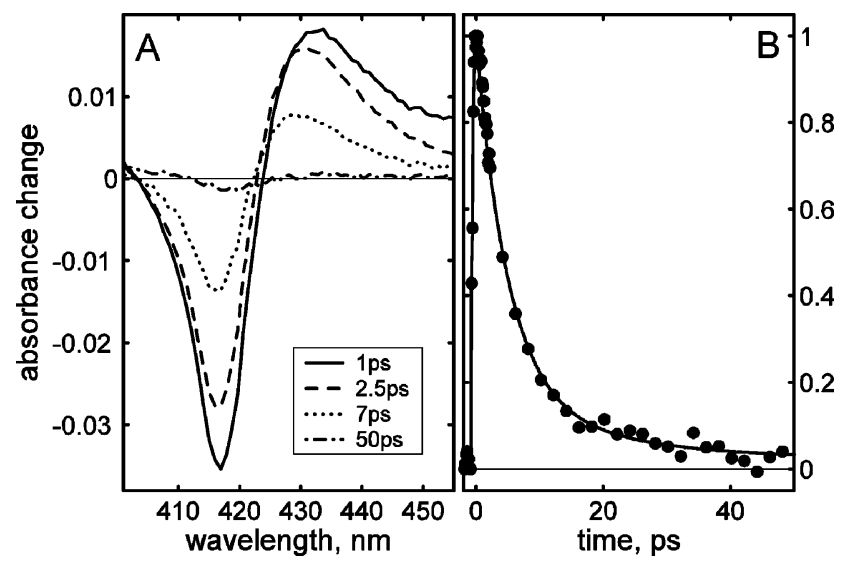

Fig. 3 Spectral evolution of the high $\mathrm{pH}(\mathrm{pH} \mathrm{8.0)}$ deoxy form of $\mathrm{cm}$ cyt. $c$. following laser excitation. Panel A: transient absorption spectra at the selected delay times after photolysis. Panel B: the kinetics at $430 \mathrm{~nm}$ (normalised to the maximum).

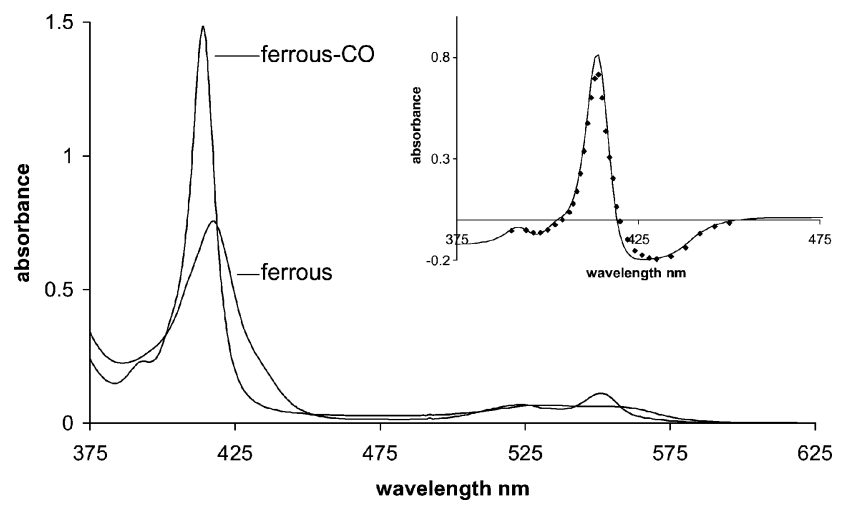

Fig. 4 The steady-state spectra of ferrous $\mathrm{cm}$ cyt. $c$ and the $\mathrm{CO}$ derivative (sodium phosphate, $20 \mathrm{mM}, \mathrm{pH}$ 7.0). Inset: comparison between the difference spectra (carbonmonoxy minus deoxy) obtained statically (solid line) and by lamp flash photolysis (solid symbols)). The protein concentration was $\sim 7 \mu \mathrm{M}$. The protein was reduced with a slight excess of sodium dithionite. The temperature was $20^{\circ} \mathrm{C}$.

of cm cyt. $c$. The two difference spectra (steady state and flash constructed) were overlaid and normalised to each other, and it was found they were virtually identical (see inset to Fig. 4). Assuming a lysine binds to the $6^{\text {th }}$ coordination position of the haem iron in the absence of CO (see Discussion section), this indicates that at the millisecond time scale of the Xenon flash experiment, a lysine coordinates to the $\mathrm{CO}$ position of the haem. This is generally consistent with the earlier attribution of a $150 \mu \mathrm{s}$ phase to lysine binding. ${ }^{4}$ Following photolysis using a xenon lamp (flash duration in the $\mu$ s range) a fraction of the photodissociated $\mathrm{CO}$ escapes to bulk solution and recombines in the ms time range at a rate dependent on the $\mathrm{CO}$ concentration. The amplitude of the absorbance change accompanying this $\mathrm{CO}$ recombination is a measure of the extent of photo-dissociation to bulk solution and has been employed to determine the apparent quantum yield, $\varphi$, using the "pulsed" method. ${ }^{1}$ The method determines the value of the $\varphi$ relative to a standard, in most incidences myoglobin, for which $\varphi \cong 1$ (i.e. one $\mathrm{CO}$ escapes to bulk solution for each photon absorbed by the haem group). The results are shown in Fig. 5 where the logarithm of the ratio of the concentrations of the $\mathrm{CO}$ adduct in the dark to that in the light (of relative intensity I) is plotted as a function of I. As predicted by theory the plots are linear passing through the origin. The slope of the plot for $\mathrm{cm}$ cyt. $c$ relative to myoglobin yields the apparent quantum yield. The value of $\varphi$ for $\mathrm{cm}$ cyt. $c$ was calculated to be 0.12 , very close to that determined by the laser flash method (see Fig. 2). This value is smaller than that previously reported $(\varphi=0.26)$ for $\mathrm{cm}$ cyt. $c .{ }^{4}$ This is the result of differences in the way in which cm cyt. $c$ was prepared.

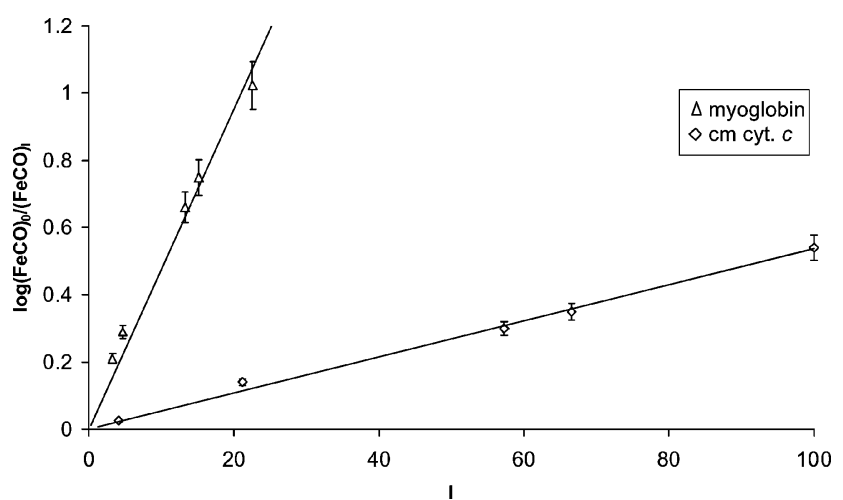

Fig. 5 Dependence of the extent of photo-dissociation of the $\mathrm{CO}$ adducts of myoglobin and cm cyt. $c$ on relative light intensity (I) expressed as a percentage of full flash intensity. The protein concentrations were $\sim 5 \mu \mathrm{M}$ dissolved in $20 \mathrm{mM}$ sodium phosphate buffer, $\mathrm{pH} 7.0,50 \mu \mathrm{M}$ $\mathrm{CO}$ and temperature $20^{\circ} \mathrm{C}$.

In particular, the preparation described here employed the minimum incubation time $(\sim 4 \mathrm{~h})$ with bromoacetic acid that is needed to fully modify the methionine- 80 residue. In earlier work incubation with the haloacid was for at least $12 \mathrm{~h}$. It is known that such lengthy incubation leads to further modification of the protein (lysine and histidine residues being susceptible) and this further disrupts the protein structure allowing easier movement of $\mathrm{CO}$ from the haem cavity, resulting in elevated apparent quantum yield.

\section{Discussion}

Following photolysis, geminate recombination of gaseous ligands in the picosecond time scale likely reflects rebinding from the immediate vicinity of the haem iron. This is in contrast to geminate recombination in the nanosecond time scale that is thought to report rebinding from within the protein matrix, and some distance from the iron. For cm cyt. $c$, analysis of the $\mathrm{CO}$ geminate process is in the picosecond time scale. This result suggests that following photolysis $\mathrm{CO}$ rebinds from within the distal pocket region, very close to the haem iron and is in or can easily adopt an orientation favourable for binding to the haem (i.e., the carbon atom pointing towards the haem iron and the $\mathrm{CO}$ axis not far from perpendicular to the haem plane). Analysis by MEM and a global model reveals that three distinct phases contribute to the geminate process. For cm cyt. $c$ then, the existence of three phases could be attributed to $\mathrm{CO}$ rebinding from three different locations from within the distal pocket region.

The explanation that multiphasic geminate $\mathrm{CO}$ recombination results from heterogeneity of the protein sample (see Materials and methods section) can be discounted for two reasons. Firstly, fractionation into separate components of $\mathrm{cm}$ cyt. $c$ prepared by long incubation with bromoacetic acid has revealed that the CO combination kinetics did not differ between these. ${ }^{3}$ Secondly, and crucially, cyt. $c$ in which the methionine- 80 ligand has been substituted by an alanine residue by site directed mutagenesis displays tri-phasic $\mathrm{CO}$ geminate recombination with time constants and spectral contributions very similar to those reported here for $\mathrm{cm}$-cyt. $c$. As the mutant protein is homogeneous we can eliminate protein heterogeneity as the cause of the complexity we observe. The results on this and several other Met80X mutants will be reported elsewhere.

However, this simple explanation needs further elaboration. Examination of Fig. 2(B) (inset) reveals that the kinetic processes exhibit different spectra, implying that $\mathrm{CO}$ recombines with a number of distinct spectral forms of the deoxy haem, i.e. with vibrationally excited species and/or forms not at structural equilibrium. For vibrationally excited, 'hot', haem species we would expect a red shift of the spectrum compared to 
thermalized haem. The finding that the induced absorption lobe of the transient spectrum red shifts in time indicates that, where haem cooling does take place on the timescale of a few $\mathrm{ps}^{18}$ this is not the main origin of the spectral evolution. Regarding the possibility of haem structural relaxations, for example, if following photo-dissociation of the $\mathrm{CO}$ molecule the haem group does not fully relax from its planar form characteristic of the liganded species, for some ps before relaxing to the fully domed configuration typical of the deoxy form, then the fastest geminate $\mathrm{CO}$ binding process may effectively compete with this molecular motion. Thus this kinetic process reports rebinding to a non-equilibrium species and displays a spectrum different from that observed to be associated with the slower geminate phases and that recorded from static spectroscopy. More generally the haem cavity of cm cyt. $c$ may allow little mobility for the dissociated $\mathrm{CO}$, which therefore initially strongly interacts with the haem, giving rise to both efficient (possibly barrierless) recombination and distortion of the haem. Competing rebinding pathways for $\mathrm{CO}$ rebinding and motion into distinct other positions can then lead to both haem relaxation and less efficient rebinding.

On rapid laser photolysis of $\mathrm{CO}$ from $\mathrm{cm}$ cyt. $c$ the fraction of $\mathrm{CO}$ that recombines by a geminate process (intramolecular) in the picosecond time scale was measured at $\sim 89 \%$, and the fraction that does not return after 4 ns was measured at $\sim 11 \%$ (see Fig. 2). The quantum yield measured by lamp flash yielded a value for $\varphi=\sim 0.12$ or, in other words, the fraction of $\mathrm{CO}$ escaping to bulk solution and recombining with the haem in a bimolecular reaction was $\sim 12 \%$. These two values are almost identical, and this would suggest that there exists no further geminate process after $4 \mathrm{~ns}$. Therefore, it appears that $\mathrm{CO}$ geminate recombination is only from within the distal pocket region close to the haem and not from further distances away located in the protein matrix itself. The close correspondence also implies that the photo-dissociation quantum yield is as high as for $\mathrm{MbCO}$, i.e. close to 1 . The $\mathrm{CO}$ geminate recombination efficiency is very high for a haem protein. In many haem proteins like myoglobin the geminate recombination amounts only to a few percent and occurs on a timescale of nanoseconds or longer. ${ }^{19}$ In other haem proteins where $\mathrm{CO}$ replaces an internal ligand substantial haem-CO rebinding has been observed, in particular in the presumed oxygen sensor $E c \operatorname{Dos}^{20}$ and in the CO-sensor CooA. ${ }^{21}$

Geminate rebinding of gaseous molecules to haem, particularly where this is multiphasic, has been used to probe the internal structure of the protein. Probably the best example of a protein where this strategy has been employed is myoglobin, with $\mathrm{NO}$ as a ligand. ${ }^{14}$

In 1965, Kendrew and co-workers discovered that Xe gas binds to myoglobin, ${ }^{22}$ and 1984 Tilton and co-workers solved the crystallographic structure of ferri myoglobin equilibrated with $\mathrm{Xe}$ revealing it contained four Xe atoms trapped in four separate cavities $\left(\mathrm{X}^{1}-\mathrm{X}^{4}\right)$ with radii $>5 \AA$ and lined with hydrophobic side chains. ${ }^{23}$ More recently, several groups have obtained the crystal structures of "intermediate states" of myoglobin that show the location $\mathrm{CO}$ following photo-dissociation. ${ }^{24,25}$ This crystallographic data shows that photo-dissociated $\mathrm{CO}$ can be seen at more than one site inside the protein matrix. The primary docking site is within the distal haem pocket, $\mathrm{CO}$ that lies parallel and $\sim 3.6 \AA$ from the haem plane. This site is occupied a few ps after photo-dissociation. ${ }^{26}$ Despite their proximity, virtually no, fast geminate $\mathrm{CO}$-haem rebinding occurs from this site, presumably at least in part because the orientation and mobility of, $\mathrm{CO}$ is unfavourable. There are two secondary sites at a greater distance from the iron, and these overlap with the Xe binding sites (namely $\mathrm{X}^{1}$ and $\mathrm{X}^{4}$ ). Brunori et al. in 2004 showed that following $\mathrm{CO}$ photo-dissociation in myoglobin, the $\mathrm{CO}$ that migrated to these cavities could then exit the protein and equilibrate with the bulk solution. ${ }^{2}$ As we do not observe any $\mathrm{CO}$ geminate process for $\mathrm{cm}$ cyt. $c$ other than in the picosecond time range that we attribute to re-binding from within the distal pocket, it would seem that $\mathrm{cm}$ cyt. $c$ does not possess such $\mathrm{CO}$ sequestering cavities that facilitate ligand escape to the exterior of the protein. It cannot be excluded, however, that the rebinding from such cavities would be as low as in $\mathrm{MbCO}$, and, given the already low probability of escape from the haem vicinity, too low to detect.

The haem iron in native cyt. $c$ is axially coordinated by two endogenous ligands, namely His 18 and Met80. It has been shown that one of these axial ligands can be photo-dissociated from the haem iron of ferrous cyt. $c$ with high yield. ${ }^{14}$ The majority of the photophysical studies on ferrous cyt. $c$ have been based on femtosecond transient absorption spectroscopy with high photon energy excitation in the Soret or UV absorption region of the haem. ${ }^{6,27}$ The main objective has been to elucidate whether the $\mathrm{Fe}-\mathrm{His}$ proximal bond or the $\mathrm{Fe}-\mathrm{Met}$ distal bond is broken on photo-excitation and conclusions have been drawn from comparison of the reconstructed photoproduct absorption spectrum with the stationary absorption spectrum. For example, Wang et al. in 2000 found a high similarity of the photoproduct absorption spectrum with the stationary absorption spectrum of a model five-coordinate His-ligated microperoxidase concluding, therefore, that the Met80 was photo-dissociated with a time constant of $\sim 6 \mathrm{ps}$ for geminate re-binding. ${ }^{6}$ However, Jongeward et al. in 1987 conducted ligand photolysis studies on cytochromes $b_{5}$ and $c$ with picosecond time resolution and concluded that the $\mathrm{Fe}-\mathrm{His}$ bond is broken with a time constant of $\sim 11$ ps for geminate recombination..$^{27}$ More recently, Cianetti et al. in 2004, working on ferrous cyt. $c$ using a novel subpicosecond Raman spectrometer/laser instrument, assigned the photoproduct as being a five-coordinate His-ligated species. ${ }^{5}$ The distinctive feature in their 1 ps spectrum of the cyt. $c$ photoproduct was the appearance of a strong $216 \mathrm{~cm}^{-1}$ band, assigned to the $\mathrm{Fe}-\mathrm{His}$ stretching. This band is not Raman active in six coordinate cyt. $c$, but is present in five coordinate ferrous high-spin domed haem structures. ${ }^{28}$ Therefore, the appearance of the Fe-His stretch indicated the photo-dissociation of an axial ligand, namely the Met80. Geminate recombination of the Met80 axial ligand was found to be with a time constant

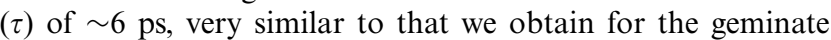
recombination of the photo-dissociated axial ligand in $\mathrm{cm}$ cyt. $c$. We believe that the process that we observe is the recombination with the haem of the lysine residue that, at high $\mathrm{pH}$, we know fills the sixth coordination position in $\mathrm{cm}$ cyt. $c$, the homologous position that Met80 occupies in the native molecule. The proximity of the amino group of lysine 79 to the haem group (Fig. 1) supports the view. We have previously shown for cm cyt. $c$ that, at elevated values of $\mathrm{pH}$, an intrinsic lysine residue binds to the sixth coordination site of the haem iron, and this is the case for both the ferrous (as studied here) and ferric forms of the protein. ${ }^{4,29}$ The apparent $\mathrm{p} K$ values for this transition were found to be $\sim 7$ and $\sim 6$ for the ferrous and ferric forms respectively, the spectra of the latter being analogous to the spectra of the alkaline form of native ferri cyt. $c .^{3,4,29}$ The close correspondence of the time constant for recombination of the internal ligand, whether it is methionine or lysine indicates that this recombination may be essentially barrierless. We note further that the fastest time constants observed for internal ligand rebinding in other sixcoordinate CO-binding haem proteins like EcDos (methionine) and the $\mathrm{CO}$-sensor CooA (proline) are very similar. ${ }^{20,21}$ However, the binding of internal residue ligands after $\mathrm{CO}$ dissociation in such protein occurs on the time scale of $\sim 100 \mu \mathrm{s}^{20,30,31}$ (see below)

An earlier estimate of the rate at which the intrinsic lysine binds to the haem of $\mathrm{cm}$ cyt. $c$ has been provided by temperature (T) jump experiments. In such experiments the "on" rate constant has been evaluated at $\sim 15000 \mathrm{~s}^{-1}$ (giving $\tau \sim 60 \mu \mathrm{s}$ ). A similar value has been reported for Met80Asp $(\tau \sim 150 \mu \mathrm{s})$ using a nanosecond laser flash method that follows events in the $\mu$ s time range following $\mathrm{CO}$ photolysis to the bulk phase. These values are clearly very different from the relaxation time 
we report here for direct lysine recombination. These differences may be reconciled by proposing that in the $T$ jump or $\mathrm{CO}$ flash experiments the lysine recombines from a location distant from the haem. This is reasonable given that in the T jump experiments the events are triggered by deprotonation of a lysine that, because of the positive charge on the amino group, is displaced outside the haem cavity. Similarly one would expect CO to displace lysine from the vicinity of the haem given the highly caged and packed nature of the haem cavity (as suggested by the low apparent quantum yield). In contrast photodissociation of a lysine already bound to the ferrous iron leads to rapid (ps) recombination, as reported here.

\section{Acknowledgements}

G. S. and M. T. W. thank the BBSRC (UK) and the European Science Foundation for financial support. We thank JeanChristophe Lambry for help with the data analysis.

\section{References}

1 M. Brunori, G. Giacometti, E. Antonini and J. Wyman, Proc. Natl. Acad. Sci. USA, 1973, 70, 3141-3144.

2 M. Brunori, D. Bourgeois and B. Vallone, J. Struct. Biol., 2004, 147, 223-234.

3 M. Brunori, M. Wilson and E. Antonini, J. Biol. Chem., 1972, 247, 6076-6081.

4 G. Silkstone, G. Stanway, P. Brzezinski and M. Wilson, Biophys. Chem., 2002, 98, 65-77.

5 S. Cianetti, M. Negrerie, M. Vos, J.-L. Martin and S. Kruglik, J. Am. Chem. Soc., 2004, 126, 13932-13933.

6 W. Wang, X. Ye, A. Demidov, F. Rosca, T. Sjodin, W. Cao, M. Sheeran and P. Champion, J. Phys. Chem., 2000, 104, 10 789-10 801.

7 Protein Electron Transfer, ed. D. S. Bendall, Bios Scientific Publishers Ltd, Oxford, 1996.

8 R. A. Marcus and N. Sutin, Biochim. Biophys. Acta., 1985, 11, 265322.

9 D. Zaslavsky, R. C. Sadoski, K. Wang, B. Durham and F. Millett, Biochemistry, 1998, 37, 14910-14916.

10 J. Moody, U. Brandt and P. Rich, FEBS Lett., 1991, 293, 102-105.
11 P. Brzezinsk, M. Sundahl, P. Ädelroth, M. T. Wilson, B. El-Agez, P. Wittung and B. G. Malmström, Biophys. Chem., 1995, 54, 191-197. 12 A. Schejter and P. George, Nature, 1965, 206, 1150-1151.

13 Y. Yao, C. Qian, K. Ye, J. Wang, Z. Bai and W. Tang, J. Biol. Inorg. Chem., 2002, 7, 539-547.

14 J.-L. Martin and M. Vos, Methods Enzymol., 1994, 232, 416-430.

15 S. W. Provencher and R. H. Vogel, Prog. Sci. Comput., 1983, 2, 304 319.

16 J. E. Morgan, M. I. Verkhovsky, A. Puustinen and M. Wikström, Biochemistry, 1985, 34, 15633-15637.

17 P. J. Steinbach, A. Ansari, J. Berendzen, D. Braunstein, K. Chu, B. R. Cowen, D. Ehrenstein, H. Frauenfelder, J. B. Johnson, D. C. Lamb, S. Luck, J. R. Mourant, G. U. Nienhaus, P. Ormos, R. Philipp, A. Xie and R. D. Young, Biochemistry, 1991, 30, 3988-4001.

18 J. W. Petrich, J.-L. Martin, D. Houde, C. Poyart and A. Orszag, Biochemistry, 1987, 26, 7914-7923.

19 M. Brunori and Q. H. Gibson, EMBO Rep., 2001, 2, 674-679.

20 U. Liebl, L. Bouzhir-Sima, L. Kiger, M. C. Marden, J.-C. Lambry, M. Négrerie and M. H. Vos, Biochemistry, 2003, 42, 6527-6535.

21 S. Kumazaki, H. Nakajima, T. Sakaguchi, E. Nakagawa, H. Shinahara, K. Yoshihara and S. Aano, J. Biol. Chem., 2000, 275, 38378 38383.

22 B. P. Shoenburn, H. C. Watson and J. C. Kendrew, Nature, 1965, 207, 28-30.

23 R. F. Tilton, I. D. Kuntz and G. A. Petsko, Biochemistry, 1984, 23, 2849-2857.

24 K. Chu, J. Vojtchovsky, B. H. McMahon, R. M. Sweet, J. Berendsen and I. Schlichting, Nature, 2000, 403, 921-923.

25 A. Ostermann, R. Waschipky, F. G. Parak and G. U. Nienhaus, Nature, 2000, 404, 205-208.

26 M. Lim, T. A. Jackson and P. A. Anfirud, Nat. Struct. Biol., 1997, 4 $422-427$.

27 K. A. Jongeward, D. Magde, D. J. Taube and T. G. Taylor, J. Biol. Chem., 1988, 263, 6027-6030.

28 T. Kitagawa, in Biological Applications of Raman Spectroscopy, ed. T. G. Spiro, Wiley, New York, 1998, vol. 3, ch. 3.

29 G. G. Silkstone, C. E. Cooper, D. Svistunenko and M. T. Wilson, J. Am. Chem. Soc., 2005, 127, 92-99.

30 J. M. Kriegl, A. J. Bhattacharyya, K. Nienhaus, P. Deng, O. Minkow and G. U. Nienhaus, Proc. Natl. Acad. Sci. USA, 2002, 99, 79927997.

31 M. Puranik, S. B. Nielsen, H. Youn, A. N. Hvitved, J. L. Bourassa, M. A. Case, C. Tengroth, G. Balakrishnan, M. V. Thorsteinsson, J. T. Groves, G. L. McLendon, G. P. Roberts, J. S. Olson and T. G. Spiro, J. Biol. Chem., 2004, 279, 21 096-21 108. 